Instituto Internacional de Investigación y Desarrollo Tecnológico Educativo INDTEC, C.A.

DOI: https://doi.org/10.29394/scientific.issn.2542-2987.2017.2.3.12.226-246

OAI-PMH: http://www.indteca.com/ojs/index.php/Revista Scientific/oai

\title{
Estrategias Didácticas Significativas para el Desarrollo de la Comprensión Lectora de los Educandos en Educación Primaria
}

\author{
Autora: María Edixa Araujo Riveros \\ Universidad Nacional Experimental Rafael María Baralt, UNERMB \\ mariaedixa9@hotmail.com \\ Zulia, Venezuela
}

\section{Resumen}

El presente artículo tiene como propósito proponer un manual de estrategias didácticas significativas para el desarrollo de la comprensión lectora en los educandos de la Unidad Educativa El Batatal ubicada en la parroquia Marcelino Briceño, municipio Baralt, estado Zulia. Para ello, se sustenta teóricamente en los aportes de autores como Campos (2011), Camilloni (2012), Castedo (2011), Ortiz (2012), entre otros. Metodológicamente, se desarrolló siguiendo la modalidad de proyecto factible, que se aplicó a una población de 24 docentes pertenecientes a la institución seleccionada. Se utilizó la encuesta como técnica la encuesta con un instrumento tipo cuestionario estructurado según escala Likert, y veinticuatro ítems. La validez se obtuvo a través del juicio de expertos, la confiabilidad de $=, 9216$ se calculó mediante el coeficiente Alfa de Cronbach. Los resultados obtenidos permitieron concluir que los docentes presentaban debilidades relacionadas con la aplicación de estrategias didácticas que posibilitaran el desarrollo de la comprensión lectora en sus educandos aportando insumos para el diseño del manual propuesto. Se recomendó utilizar estrategias diversas para los diferentes niveles de comprensión lectora de acuerdo con el desarrollo alcanzado por sus educandos.

Palabras clave: estrategias didácticas significativas; comprensión lectora; educación primaria. 


\title{
Teaching Strategies for Significant Development of Reading of Learners Understanding in Primary Education
}

\begin{abstract}
This article aims to propose a manual significant for the development of reading comprehension in students of Educational Unit The Batatal located in the parish Marcelino Briceño, municipality Baralt, Zulia state teaching strategies. To do this, it is based theoretically on the contributions of authors like Campos (2011), Camilloni (2012), Castedo (2011), Ortiz (2012), among others. Methodologically, it developed following the modality of feasible project, which was applied to a population of 24 teachers from the selected institution. survey the survey with a structured according Likert scale questionnaire type instrument, and twenty-four items was used as a technique. The validity was obtained through expert judgment, the reliability of $=, 9216$ was calculated using Cronbach's alpha coefficient. The results led to the conclusion that teachers had weaknesses related to the implementation of teaching strategies that will enable the development of reading comprehension in students by providing input for the design of the proposed manual. It is recommended to use different strategies for different levels of reading comprehension in accordance with the development achieved by their students.
\end{abstract}

Keywords: meaningful teaching strategies; reading comprehension; primary education.

Date Received: 31-08-2016

Date Acceptance: 19-09-2016 


\section{Introducción}

En datos recientes aportados por la Organización de las Naciones Unidas para la Educación, la Ciencia y la Cultura, el número de analfabetos adultos en América Latina asciende a 30 millones de personas, cifra que no incluye, según Rivero (2012: 18) a "los analfabetos funcionales, resultado de la ausencia de estrategias didácticas dirigidas a la formación de lectores independientes y personas que sepan realmente escribir y, no sólo reproducir textos escritos".

Estas analfabetas funcionales son los adultos que egresaron de la escuela sin saber realmente hacer uso de la lengua escrita; aun cuando una de las finalidades de la educación es formar lectores independientes e individuos productores de textos escritos, de carácter funcional y artístico. Esta realidad hace necesario que el proceso de enseñanza de la lectura se desarrolle en un ambiente donde se propicie la participación activa y crítica de los estudiantes.

Lo anterior implica cambiar las concepciones actuales, las cuales se ubican entre dos enfoques: el que confunde leer con descifrar, escribir con copiar y donde la actividad de la clase se organiza en torno a una secuencia de lecciones que deben aprenderse a través de la ejercitación mecánica, memorización y copia sistemática del modelo activista en el cual no se explotan todas las potencialidades de los educandos a favor de un respeto mal entendido a su proceso de aprendizaje, olvidando el carácter intencional de la enseñanza y necesaria orientación del docente.

Por lo antes expuesto, la presente investigación se dirigió a proponer un manual de estrategias didácticas significativas para el desarrollo de la comprensión lectora en los educandos de la Unidad Educativa El Batatal ubicada en la parroquia Marcelino Briceño, municipio Baralt, estado Zulia. 


\section{Teoría y Conceptos}

\subsection{Objetivos de la Investigación}

En la presente investigación se plantearon los siguientes objetivos, señalados a continuación:

\subsubsection{Objetivo General:}

Proponer un manual de estrategias didácticas significativas para el desarrollo de la comprensión lectora en los educandos de la Unidad Educativa El Batatal ubicada en la parroquia Marcelino Briceño, municipio Baralt, estado Zulia.

\subsubsection{Objetivos Específicos:}

Describir el proceso de aprendizaje de la lectura de los niños y niñas de los tres primeros grados de la Unidad Educativa El Batatal ubicada en la parroquia Marcelino Briceño, municipio Baralt, estado Zulia.

Identificar las estrategias didácticas que utilizan los docentes para el aprendizaje de la lectoescritura de los niños y niñas de los tres primeros grados de la Unidad Educativa El Batatal ubicada en la parroquia Marcelino Briceño, municipio Baralt, estado Zulia.

Diseñar un manual de estrategias didácticas significativas para el desarrollo de la comprensión lectora en los educandos de la Unidad Educativa El Batatal ubicada en la parroquia Marcelino Briceño, municipio Baralt, estado Zulia.

2.2. Las estrategias didácticas: son las formas en que el docente crea una situación que permita al educando desarrollar la actividad de aprendizaje, es decir, hacer referencia a una acción planeada especialmente para una situación concreta. Al respecto, Fairstein y Gyssels (2010), indica que una estrategia didáctica puede planificarse para una clase o una serie de ellas, 
especificando la forma de organizar el grupo, el orden de presentación de los contenidos, la preparación del ambiente de aprendizaje, el material didáctico a utilizar, las actividades a realizar por los estudiantes y el tiempo para cada una de ellas.

Existen diversas estrategias y la variedad depende de la creatividad del educador, por tanto, es recomendable combinar y articular diferentes estrategias de enseñanza a lo largo del tratamiento de un contenido, en diferentes clases o dentro de un aula. En este marco de ideas, Feldman (2011), propone una serie de recomendaciones para la selección y preparación de estrategias didácticas para que éstas sean significativas para los estudiantes.

Para planificar las estrategias se debe organizar un esquema o mapa del contenido que quiere enseñar a trabajar con los estudiantes. Esto permite a los docentes analizar posibles actividades, por cuál tema comenzar, cuántas clases va a necesitar para los distintos temas, entre otros aspectos.

Al respecto, Díaz y Hernández (2010: 162), definen las estrategias didácticas como "los procedimientos o recursos utilizados por el agente de enseñanza para promover aprendizajes significativos". De lo anterior se deduce que las estrategias tipifican y ordenan las actividades docentes para el logro del aprendizaje significativo de los educandos en las diferentes áreas académicas, concretamente en el desarrollo de la comprensión lectora.

De allí que autores como Solé (2011) y Alonso (2012), señalen que las estrategias significativas para la enseñanza de la lectura, deben propiciar desde el principio la comprensión del texto, la cual es una actividad compleja, constructiva, pues durante el proceso el aprendiz trata de construir, una representación a partir de los significados sugeridos por el texto, para lo cual utiliza todos sus recursos cognitivos pertinentes, como esquemas, habilidades, estrategias. 
Esta construcción se elabora a partir de la información que le propone, pero esta se ve fuertemente enriquecida por las interpretaciones, inferencias, integraciones que el lector adiciona con la intención de lograr una representación fiel de lo que el autor quiso comunicar. Al respecto, Ortiz (2012), propone entre otras, las siguientes estrategias significativas:

Crear un ambiente propicio para el autoconocimiento, la autorreflexión, el discernimiento, la cooperación y el diálogo; vivenciando espacios donde se haga uso del lenguaje oral y escrito, leyendo textos reales en contextos similares a la práctica social, lo cual se puede lograr mediante las siguientes acciones:

1. Creación de espacios para la lectura de textos de diversos géneros discursivos en contextos funcionales y comunicativos: de información científica, periodísticos, relatos históricos, literarios, humorísticos, poesías, obras de teatro, instruccionales, publicitarios, de acuerdo al nivel cognoscitivo de los/as estudiantes. Es conveniente favorecer la exploración y el descubrimiento guiado de la computadora como recurso tecnológico, mediante el uso de hipertextos que posibilitan la interacción texto-imagen-sonido.

Para ello, el docente debe proponer en el aula, incluso desde la educación inicial, actividades que lleven al niño/a a la búsqueda de significados en el texto escrito, pues de esta manera, el educando descubrirá la relación existente entre el lenguaje y la escritura, enfrentándose a retos, buscando soluciones al problema de interpretar textos, los cuales representen el lenguaje real, no una serie de sonidos, sílabas y palabras aisladas.

2. Creación de espacios para la confrontación de las diferentes interpretaciones de un texto, de manera que los/as estudiantes confirmen y amplíen sus anticipaciones o descubran sus contradicciones, argumentando sus puntos de vista y buscando pistas en el texto por medio de la relectura del texto o mediante el uso de imágenes existentes en el mismo. 
Lo anterior implica para favorecer la comprensión lectora, meta primordial de la lectura, el docente debe propiciar en el aula la discusión y justificación de las diferentes interpretaciones de un mismo texto, de manera que los estudiantes confirmen sus anticipaciones o descubran sus contradicciones, confrontando diferentes puntos de vista, buscando índices en el texto a través de la relectura del mismo.

3. Creación de espacios para que los/as estudiantes y el docente confronten las estrategias de lectura utilizadas, con el propósito de fomentar la autorreflexión y avanzar en el proceso de comprensión lectora.

Ofrecer al estudiante experiencias de interacción con el lenguaje escrito, mediante las siguientes acciones sistemáticas:

1. Creación de un ambiente alfabetizador con la presencia de textos de diferentes géneros discursivos al alcance de los participantes, tales como el rincón de la lectura, bibliotecas de aula, periódicos murales y escolares, carteleras informativas, entre otros.

2. Espacios para la lectura con diferentes intencionalidades y en situaciones lo más parecidas a la práctica social, estas actividades se deben realizar en diferentes situaciones, aun si el/la niño/a no lee convencionalmente. En esta estrategia, el docente además de leer para los estudiantes, organiza espacios donde ellos exploren el material escrito, simulando hacer uso de la lectura, realizando predicciones, anticipaciones e inferencias apoyándose en sus conocimientos previos y en las pistas proporcionadas por el texto.

De igual modo, Díaz y Hernández (2010): señalan algunos tipos de estrategias que pueden ser enseñadas a los estudiantes para el aprendizaje de la lectura: previas a la lectura, durante la lectura y después de la lectura.

Estrategias previas a la lectura: según los autores antes mencionados, éstas comprenden todas las que se plantean preferentemente antes de llevar 
a cabo el proceso, las cuales tienen que ver con el establecimiento del propósito para leer y con las actividades de planeación sobre cómo enfrentar el proceso de comprensión de la lectura utilizando los recursos cognitivos disponibles.

De acuerdo con lo anterior, se infiere que el educando al momento de iniciar el proceso de aprendizaje de la lectura, tiene un propósito previo, el cual es individual, y para ello, utiliza los conocimientos previos adquiridos en el hogar o el grado anterior. Dentro de ellas, los autores proponen: usar el conocimiento previo, elaborar predicciones, plantear preguntas relevantes.

Estrategias durante la lectura: para Díaz y Hernández (2010), estas estrategias son las que se aplican cuando ocurre la interacción directa con el texto y cuando se estén ejecutando los micro y macroprocesos de lectura. Entre ellas mencionan: las inferencias y el uso de estructuras textuales.

Estrategias después de la lectura: ocurren cuando ya ha tenido lugar la actividad de lectura o cuando ha finalizado parte de la misma. Díaz y Hernández (2010: 152) señalan que "las estrategias típicas realizadas después de finalizar un acto de comprensión son dos variantes de la atribución del sentido conseguidas gracias a la interacción entre los conocimientos previos y las características del texto: identificación de la idea principal y el resumen".

Estas estrategias son actividades cognitivas pertenecientes al procesamiento macroestructural del texto. La identificación de la idea principal requiere de la realización de tres pasos: (a) comprender lo leído; (b) hacer juicios sobre la importancia de la información; (c) consolidar la información. Esta estrategia es difícil para los/as estudiantes de los primeros grados, pues depende del tipo de texto y de sus conocimientos previos sobre el contenido temático del mismo. 
2.3. Estrategias previas a la lectura: según Díaz y Hernández (2010), éstas comprenden todas las que se plantean antes de llevar a cabo el proceso, las cuales tienen que ver con el propósito del estudiante y la planificación sobre cómo enfrentar dicho proceso utilizando los recursos cognitivos disponibles. Dentro de estas estrategias proponen:

1. Usar el conocimiento previo pertinente para facilitar la atribución de significado del texto.

2. Elaborar predicciones acerca de lo que tratará el texto.

3. Plantear preguntas relevantes sobre el tema.

2.4. Estrategias durante la lectura: Díaz y Hernández (2010) señalan que éstas se aplican cuando ocurre la interacción directa con el texto y cuando se están ejecutando los micro y macroprocesos de lectura, mencionando entre ellas las inferencias y el uso de estructuras textuales.

2.5. Estrategias después de la lectura: ocurren cuando ya culmina la actividad de lectura o parte de la misma. Son actividades cognitivas pertenecientes al procesamiento macro estructural del texto. Entre ellas se ubican la identificación de la idea principal y el resumen.

\section{Metodología}

Dicho artículo, metodológicamente se desarrolló siguiendo las pautas de un estudio de campo, no experimental, transeccional enmarcado en un proyecto factible, aplicado a una población de 24 docentes a quienes se suministró un instrumento tipo cuestionario con cinco alternativas de respuesta y 18 ítems, validado por expertos y alcanzando 0.9216 como coeficiente de confiabilidad obtenido por Alfa Cronbach. 


\subsection{Tipo de investigación}

El tipo de investigación se enmarca en un proyecto factible por cuanto el objetivo general del estudio determina la investigación a realizar, siendo en este caso proponer un manual de estrategias didácticas significativas para el desarrollo de la comprensión lectora en los educandos de la Unidad Educativa El Batatal ubicada en la parroquia Marcelino Briceño, municipio Baralt, estado Zulia.

Los proyectos factibles son aquellas investigaciones que proponen o sugieren soluciones prácticas un determinado problema, fundamentándose en trabajos de campo, documentales o una combinación de ambos, sustentándose en lo planteado por la Universidad Pedagógica Experimental Libertador (2012: 6): quien lo define como “...la investigación, elaboración y desarrollo de una propuesta de un modelo operativo viable para solucionar problemas, requerimientos o necesidades de organizaciones o grupos sociales; puede referirse a la formulación de políticas, programas, tecnologías, métodos o procesos...".

Al tener como modalidad el proyecto factible, se estructura el trabajo en las siguientes fases: un diagnóstico de la situación existente en la Unidad Educativa El Batatal ubicada en la parroquia Marcelino Briceño, municipio Baralt, estado Zulia, en cuanto a las estrategias didácticas significativas para el desarrollo de la comprensión lectora en los educandos, con el propósito de determinar los requerimientos para generar un modelo de competencias docentes, el cual se llevará a cabo mediante la aplicación de un instrumento relacionado con los tópicos de estudio.

La segunda fase corresponde a la factibilidad, donde se establecen los criterios que permiten asegurar el uso óptimo de los recursos empleados, así como los efectos del proyecto en el área o sector al cual se destina a identificar las estrategias didácticas que utilizan los docentes para el aprendizaje de la lectoescritura de los niños y niñas de los tres primeros grados de la Unidad 
Educativa El Batatal ubicada en la parroquia Marcelino Briceño, municipio Baralt, estado Zulia.

La tercera fase se realizará, de acuerdo con los resultados obtenidos, del análisis de la información, en ella se diseñará un manual de estrategias didácticas significativas para el desarrollo de la comprensión lectora en los educandos de la Unidad Educativa El Batatal ubicada en la parroquia Marcelino Briceño, municipio Baralt, estado Zulia.

En esta fase se especifica el propósito, destinatarios, la justificación de la propuesta, así como los fundamentos teóricos para su sustentación, los cuales se incluirán a manera de presentación, donde se expondrán las corrientes del pensamiento y teorías que la validan.

Finalmente, la quinta fase se refiere a la evaluación de la propuesta, en donde se valorará tanto el proceso como los resultados. Por tanto, implica diagnóstico, factibilidad, diseño y ejecución. En ella se detallarán los siguientes aspectos: a) planeación del diagnóstico; b) metodología para determinar la factibilidad; c) el proceso de diseño; d) ejecución; e) resultados obtenidos en cada paso atendiendo a los objetivos planteados.

\subsection{Diseño de la investigación}

La presente investigación se realizará sobre la base de un diseño no experimental y sin control de variables, en el contexto de un estudio de campo, pues la información obtenida como producto de la recolección de las respuestas emitidas por la población por parte de la investigadora tendrá un carácter primario, debido a que será recogida en el escenario donde se desarrolla la problemática objeto de estudio relacionada con la evaluación de un manual de estrategias didácticas significativas para el desarrollo de la comprensión lectora en los educandos de la Unidad Educativa El Batatal ubicada en la parroquia Marcelino Briceño, municipio Baralt, estado Zulia. 
En este sentido, Díaz y Hernández (2010: 158) expresan en torno al carácter no experimental del estudio que estos "se realizan sin manipular deliberadamente las variables... En un estudio no experimental no se construye ninguna situación, sino que se observan situaciones ya existentes". De igual modo, Sabino (2008: 89) en relación con el trabajo bajo el contexto del estudio de campo señala:

Los diseños de campo son los que se refieren a los métodos a emplear cuando los datos de interés se recogen de forma directa de la realidad mediante el trabajo concreto del investigador y su equipo, estos datos obtenidos directamente de la experiencia empírica, son llamados primarios, denominación que alude al hecho de que son datos de primera mano, originales, producto de la investigación en curso sin intermediación de alguna naturaleza.

\subsection{Población}

Para el desarrollo de todo proyecto sustentado en una investigación de campo, es necesario contar con los elementos necesarios de los cuales se obtendrá la información que se pretende recolectar. En ese sentido, Chávez (2007: 162) señala "la población de un estudio es el universo de la investigación, sobre el cual se pretende generalizar los resultados". Por tanto, la población para el presente estudio estará conformada por 24 docentes de Unidad Educativa El Batatal ubicada en la parroquia Marcelino Briceño, municipio Baralt, estado Zulia.

\subsection{Muestra}

Una vez delimitada la población de estudio, se debe seleccionar una muestra que sea un reflejo fiel del conjunto de la misma, definida por Balestrini (2009: 142) como "una parte representativa de una población, cuyas características deben reproducirse en ella, lo más exactamente posible". 
En el nivel de determinación de la muestra, este procedimiento se obviará dada la poca cantidad de sujetos conformantes de la población, lo que determina trabajar con la totalidad de la misma, es decir, se consideró el censo poblacional, pues estadísticamente será manejable a los efectos de la aplicación del instrumento de recolección de información. Esta decisión se sustenta en lo planteado por Chávez (2007: 166) cuando señala "las poblaciones pequeñas deben tomarse en su totalidad para garantizar la consecución de datos confiables para el estudio".

\subsection{Técnica e instrumento de recolección de información}

Todo investigador, debe confrontar con la realidad la teoría formulada y de alguna manera obtener el registro de los datos provenientes del objeto de estudio, surgiendo entonces la necesidad de su recolección, lo cual involucra dos condiciones: la técnica para la recolección de los datos, así como el instrumento utilizado para realizar dicha actividad.

La técnica utilizada en el presente estudio será la encuesta por considerar que la misma podía usarse para recabar datos vinculados con las opiniones de la población en torno a la frecuencia con que se presenta el fenómeno investigado relacionado con la relación entre las estrategias didácticas significativas para el desarrollo de la comprensión lectora en los educandos de la Unidad Educativa El Batatal ubicada en la parroquia Marcelino Briceño, municipio Baralt, estado Zulia. Al respecto, Finol y Camacho (2012: 69) expresan "la encuesta es una herramienta utilizada por el sujeto investigador para recabar información acerca del hecho, evento o fenómeno que investiga".

Asimismo, Sabino (2008: 71) señala que la encuesta trata de una técnica para "requerir información a un grupo socialmente significativo de personas acerca de los problemas en estudio para luego, mediante un análisis de tipo cuantitativo, sacar las conclusiones que se correspondan con los datos 
recogidos". Con respecto al instrumento de recolección de información se implementará un cuestionario definido por Balestrini (2009: 138) como: ...un medio de comunicación escrito y básico entre el encuestador y el encuestado, facilita traducir los objetivos y las variables de la investigación a través de una serie de preguntas muy particulares, previamente preparadas de forma cuidadosa susceptibles de analizar en relación al problema estudiado.

Bajo tales perspectivas, en la presente investigación se utilizará la modalidad del cuestionario simple, el cual es aquel en el que los encuestados mediante previa lectura, respondan por escrito sin intervención directa del investigador. En tal sentido, el investigador diseñará el instrumento, uno para medir ambas variables. Para su elaboración se aplicarán los siguientes procedimientos: se partirá de la lectura del soporte teórico de cada variable para posteriormente operacionalizarlas en sus dimensiones e indicadores respectivos.

A continuación, se elaborará el banco de ítems para cada instrumento para luego evaluar cada reactivo desechando los repetidos, confusos o no pertinentes con el indicador, cada ítem estará conformado por cinco alternativas de respuestas: siempre (S), casi siempre (CS), algunas veces $(\mathrm{AV})$, casi nunca $(\mathrm{CN})$, nunca $(\mathrm{N})$ y 18 ítems, los cuales se ubicarán en el instrumento para considerar las opiniones emitidas por la población participante a las afirmaciones propuestas en el mismo.

\subsection{Validez}

El instrumento por ser el medio material que se emplea para recoger y almacenar la información, debe ser sometido a los procesos de validez y confiabilidad. La validez es una de las pruebas de gran relevancia debido a que de esta forma se comprueba si la variable, dimensiones e indicadores son 
medidos por el cuestionario, en busca de la adecuación o exactitud suficiente y satisfacer los indicadores de las preguntas.

La validez, según Finol y Camacho (2012: 82) "constituye el proceso mediante el cual se estima: a) la medida en que las conclusiones representan la realidad empírica, b) si los constructos elaborados por los investigadores representan las categorías reales de la experiencia humana". Con base en lo anterior, el cuestionario de esta investigación será sometido a un proceso de validación de contenido mediante el juicio de expertos, quienes lo revisarán en cuanto a forma y contenido para considerar la pertinencia del mismo con los objetivos de la investigación.

A tal efecto se elaborará un diseño de validación de instrumentos de recolección de información el cual se le entregará a cinco (5) especialistas para que realicen su lectura, corrección del instrumento a aplicar, a los efectos de cumplir con la validación del mismo y arrojar las observaciones emitidas con el objeto de efectuar las correcciones necesarias y proceder a aplicarlo.

\subsection{Confiabilidad}

La confiabilidad se refiere al grado en que la aplicación repetida del instrumento al mismo sujeto $u$ objeto, produce los mismos resultados, definida por Landeau (2007: 81) "como el grado con el cual el instrumento prueba su consistencia, por los resultados que produce al aplicarlo repetidamente al objeto de estudio". Para la presente investigación se aplicará el Coeficiente Alfa de Cronbach, el cual se utiliza cuando el instrumento tiene varias alternativas de selección para responder, siendo este representado mediante la fórmula:

$$
\mathrm{rtt}=\mathrm{k} / \mathrm{k}-1\left[1-\sum \mathrm{Si}^{2} / \mathrm{St}^{2}\right]
$$

donde:

$$
\begin{aligned}
& k=\text { número de ítems } \\
& 1=\text { constante }
\end{aligned}
$$


$\mathrm{Si}^{2}=$ Varianza de los ítems

$\mathrm{St}^{2}=$ Varianza de los totales

Al operacionalizar la fórmula anterior, mediante el paquete estadístico SPSS versión 12.0, con los datos aportados por la aplicación de una prueba piloto, a una población con características similares a la del estudio se obtendrá un cociente que debe ubicarse entre 0.6 y 1 , el cual indicará que el instrumento es confiable y puede ser aplicado.

\subsection{Procesamiento de los datos}

El método de investigación seleccionado debe estar acorde con el tipo de investigación que se realiza, lo cual implica el uso de la estadística descriptiva en la presente investigación para obtener una visión global de todo el conjunto de datos, los cuales se registrarán en gráficos, donde se indicará la frecuencia porcentual de las dimensiones e indicadores

Para la tabulación de los datos se utilizará una tabla de doble entrada donde se vaciarán los datos de las variables con sus respectivos indicadores e ítems, colocando en el margen izquierdo el número de sujetos participantes en el estudio, conformados por docentes de la Unidad Educativa El Batatal ubicada en la parroquia Marcelino Briceño, municipio Baralt, estado Zulia.

En las columnas que formarán las tablas se colocarán los puntajes por ítem, de acuerdo con las alternativas seleccionadas por la población participante. Luego se utilizarán los totales presentes en la matriz de tabulación en matrices convenientes, en las cuales se colocarán los estadísticos resultantes del procesamiento matemático de los datos aportados por la población objeto de estudio.

La medición de las variables se efectuará mediante la aplicación de cálculos de estadística frecuencial para obtener las frecuencias absolutas y relativas de los datos aportados por la población, como una forma de 
evidenciar la manifestación de los indicadores en el comportamiento del sistema de variables del trabajo.

\section{Discusión}

La presente investigación se planteó como objetivo general, Proponer un manual de estrategias didácticas significativas para el desarrollo de la comprensión lectora en los educandos de la Unidad Educativa El Batatal ubicada en la parroquia Marcelino Briceño, municipio Baralt, estado Zulia, obteniéndose como resultado que los docentes implementan pocas estrategias para motivar a los educandos a perseguir la consolidación del proceso cónsono en la lectura como fuente de crecimiento intelectual y cognitivo, por tanto, el personal de la educación no sólo deben poseer los conocimientos necesarios para un desempeño laboral eficaz, sino que también, deben idear herramientas que les permitan de manera rápida y sencilla instruir a los niños y niñas en las competencias de aprendizaje integral. Asimismo, es indispensable la capacidad técnica para hacer buen uso de los recursos y el conocimiento en un área específica.

Los directivos siempre manifiestan sus opiniones de manera adecuada, asumiendo que por falta de monitoreo no es que se presenta esto, a veces, los docentes no asumen con un grado óptimo de responsabilidad lo que se les confiere en su quehacer diario para con el proceso de enseñanza aprendizaje de los estudiantes.

\section{Resultado}

Los resultados señalaron que el $85 \%$ de los docentes utilizan las estrategias tradicionales de silabeo, decodificación, lectura silenciosa, dependiendo del grado donde se desempeñan; el 24\% utilizan incipientemente las inferencias, sobre todo con la ayuda de secuencia de imágenes, en las que 
el educando debe narrar la historia para luego comparar sus inferencias con el texto escrito.

En relación a las actividades posteriores a la lectura, el $92 \%$ de los docentes utiliza la copia y el dictado como actividades predominantes, por cuanto todos los estudiantes usan el mismo texto de lectura, lo que facilita al docente dichas actividades y el $8 \%$ se declinan por tomar lectura individualmente en el pupitre o mesa.

Estos resultados indicaron debilidades en los docentes relacionados con la aplicación de estrategias significativas para el desarrollo de la comprensión lectora en los tres primeros grados de educación primaria, lo cual aportó insumos para el diseño de la propuesta, conformada por un manual donde se describen diversas estrategias para los diferentes momentos de la lectura y niveles de comprensión lectora.

Por otro lado, los resultados obtenidos permitieron a la investigadora formular las siguientes recomendaciones destinadas a optimizar la situación presentada:

Estimular la participación y compromiso de los docentes promoviendo el liderazgo en todos los niveles, así como el desarrollo de sus habilidades y conocimientos, desplegando las mejores prácticas pedagógicas a través de un proceso comunicacional asertivo, para propiciar la excelencia en su desempeño docente, el cual no se limita al aspecto de estrategias innovadoras, sino que abarca el ámbito de la enseñanza integral.

Propiciar en el aula con los educandos un ambiente de trabajo productivo, tanto en el área de lenguaje como en las demás; siempre estimulando al estudiantado a seguir estrategias que les incentiven a promover el proceso de lectura desarrollando actitudes positivas hacia la excelencia académica a través del diálogo y la confrontación constructiva de ideas.

Realizar círculos de reflexión interactivo (Lectura de cuentos y otros) que conduzcan a construir un horizonte colectivo donde converjan todos los 
docentes como equipo de trabajo, lo cual propiciará la excelencia en el desempeño de éstos, al sentir que son tomados en cuenta, de manera más activa.

Ofrecer apoyo individualizado a los niños y niñas, al momento de realizar el acompañamiento pedagógico, para contribuir en su proceso de formación académico.

Facilitar el aprendizaje de los estudiantes, incorporando a la planificación de nuevos enfoques pedagógicos que hagan de sus aprendizajes un enlace novedoso y satisfactorio.

\section{Conclusiones}

Con respecto al primer objetivo específico se determinó que el proceso de aprendizaje de la lectura de los niños y niñas de los tres primeros grados de la Unidad Educativa El Batatal, es netamente memorístico, donde la lectura se concibe como habilidades que pueden enseñarse de forma independiente, pues su enseñanza sigue siendo netamente tradicional, con deletreo, silabeo, copia, formación de palabras, entre otros.

En relación al segundo objetivo, se evidenció que las estrategias utilizadas por los docentes para la enseñanza de la lectoescritura se remiten a la descomposición de palabras en sonidos elementales, ejercicios aislados de letras, copia, caligrafía y/o dictado de las palabras aprendidas, lo que solo favorece la decodificación y la memorización.

Por esa razón, se diseñó un manual de estrategias significativas que posibilite el desarrollo de la comprensión lectora de los niños y niñas de los tres primeros grados de la Unidad Educativa El Batatal, parroquia Marcelino Briceño, municipio Baralt, estado Zulia. 


\section{Referencias}

Alonso, T. (2012). El proceso de lectura: de la teoría a la práctica. Editorial La Muralla. Madrid.

Balestrini, M. (2009). Cómo se elabora el proyecto de investigación. Editorial Consultores Asociados. Caracas.

Camilloni, S. (2012). Lecturas recreativas y la educación. Editorial Ariel. Barcelona, España.

Campos, L. (2011). Competencias y competitividad creativa. Diseño para organizaciones latinoamericanas. Editorial McGraw-Hill. Bogotá.

Castedo, P. (2011). Literatura infantil del aprendizaje. Fondo Editorial FEDUPEL. Caracas.

Chávez, N. (2007). Introducción a la investigación educativa. Editorial Gráfica González. Maracaibo.

Díaz, F. y Hernández, G. (2010). Estrategias docentes para un aprendizaje significativo. Editorial McGraw-Hill. México.

Fairstein, C. y Gyssels, M. (2010). ¿Cómo se enseña? Formación Pedagógica. Colección Programa Internacional de Formación de Educadores Populares. Fe y Alegría. Caracas.

Feldman, R. (2011). Aprender a enseñar. Editorial Aique. Buenos Aires.

Finol, M., y Camacho, H. (2012). "El Proceso de Investigación Científica". Editorial Ediluz. Maracaibo.

Landeau, R. (2007). Elaboración de Trabajos de Investigación. Editorial Alfa. Caracas.

Ortiz, B. (2012). El aprendizaje y la enseñanza de la lectura y la escritura. Colección Procesos Educativos. Fe y Alegría. Caracas.

Rivero, C. (2012). El arte y la pedagogía del conocimiento. Editorial McGraw-Hill. Bogotá.

Sabino, C. (2008). El proceso de la Investigación. Editorial Panapo. Caracas.

Solé, N. (2011). Estrategias de comprensión de la lectura. Editorial Aique. Buenos Aires.

Universidad Pedagógica Experimental Libertador (2012). Manual de Trabajos de Grado de Especialización y Maestría y Tesis Doctorales. Caracas: Autor. 


\section{María Edixa Araujo Riveros \\ e-mail: mariaedixa9@hotmail.com}

Nació en Pueblo Nuevo, Municipio Baralt, Estado Zulia, Venezuela. El 09 de Abril de 1976. Estudiante de la Universidad Nacional Experimental "RAFAEL MARÍA BARALT" en el DOCTORADO EN EDUCACIÓN. Estudios de postgrado: Universidad Nacional Experimental "RAFAEL MARÍA BARALT", título obtenido MSc. En Administración de la Educación Básica. Estudio de Pregrado: U.N.E.R.M.B; título Obtenido: LICENCIADA EN EDUCACIÓN MENCIÓN EDUCACIÓN INTEGRAL. Ha participado en cursos, talleres y simposios sobre Nuevo Enfoque de Educación Básica. UNERMB. Mapas Mentales y Conceptuales. Instrumentos de Evaluación Cualitativa. Para la I y II de Educación Básica. III Jornadas de Docentes Investigadores en Acción. Normas de Presentación de Trabajo Científico. La Planificación Estratégica como Herramienta de Integración Escuela- Comunidad. El Rol del Gerente para la Integración de los Padres y Representantes. Experiencia laboral: Escuela Básica Municipal "El Boquete", Sector El Boquete - Baralt, Zulia docente de aula (2 años). Escuela Básica Concentrada Estadal "Santa Elena" creación NER N²16, Sector Santa Elena Municipio Andrés Bello - Trujillo, docente de aula (3 años). Escuela Básica Concentrada "Don Rafael Briceño", Sector La Golfa - Baralt Zulia (5 años), docente de aula. Facilitadora de la Misión Ribas en el ambiente El Batatal (2 años). CEIB Don José Mariano Núñez de Cáceres, El Tigre Baralt; Zulia, cargo coordinadora del PAE (7 AÑOS). Docente universitario en la UBV Misión Sucre en la aldea Dr. Jesús María Portillo Anexo El Batatal (6 años). U.E.N. "El Batatal", NER 465 docente de CRA desde febrero 2015 hasta la fecha. 